\title{
A case of vasospastic angina in the detection of a high signal intensity area by using balanced SSFP with inversion recovery
}

\begin{abstract}
Image evaluation for vasospastic angina frequently generally utilizes nuclear medicine or cardiac magnetic resonance (CMR). T2-weighted image by CMR particularly is a non-invasive and simple evaluation method with some issues. Those issues might often be an unclear boundary area for high signal intensity, poor visualization due to myocardial saturation by arrhythmia, and difficulty in evaluation.

In the present study, we reported a case in the clear detection of a high signal intensity area compared to the existing T2-weighted image while concurrently utilizing "Inversion Recovery" and "Balanced SSFP (steady-state free precession)" as usable even with a general-purpose equipment for solving the above-mentioned issues
\end{abstract}

Volume 3 Issue 6 - 2017

\section{Kazue Matsumoto}

Department of Radiological Technology, Shizuoka City Hospital, Japan

Correspondence: Kazue Matsumoto, Department of Radiological Technology, Shizuoka City Hospital, Japan, Email matsumotokazue@gmail.com

Received: July 07, 2017 | Published: August 07, 2017

Keywords: CMR, arrhythmia, myocardial, myocardial infarction

Abbreviations: CMR, cardiac magnetic resonance; DM, diabetes mellitus; IHD, ischemic heart disease; VT, ventricular tachycardia; ECG, electrocardiogram; emCAG, emergency coronary angiography; STEMI, st-segment elevation myocardial infarction; CAG, coronary angiography; EF, ejection fraction; BMIPP, $\beta$-methyliodophenyl pentadecanoic Acid; ACS, acute coronary syndrome; LAD, left anterior descending coronary artery

\section{Introduction}

It is said that vasospastic angina in Japan can be approximately 3 times higher than Europe/the US. Furthermore, it is also said that approximately $80 \%$ of angina pectoris and myocardial infarction may be related to coronary vasospasm and also would cause a sudden death. However, since it is difficult to detect vasospastic angina under the electrocardiogram change, we frequently make diagnosis by T2-weighted image using BMIPP, MIBG, CMR with use of nuclear medicine as an ischemic memory. The usage of quantitative evaluation by T1/T2 mapping has recently been increasing for myocardial evaluation by CMR other than T2-weighted image. Yet, these evaluation methods are not generally common due to the requirement of special sequence or software. Thus, we have attempted to visualize edema caused by ischemia with concurrently use of Inversion Recovery and Balanced SSFP as usable even with a generalpurpose equipment.

\section{Case}

\section{Male at the age of 79}

Medical history: High blood pressure Diabetes mellitus (DM)

No allergy, hypersensitiveness, and asthma

Personal history: History of alcohol intake Daily drinker.

History of smoking: Currently non-smoker, but used to smoke 15 sticks/day until 20-21years old

\section{Current medical history}

Because the patient felt a sense of discomfort in the left chest at dawn approximately 6 months ago, he visited another clinic. Later, he had a follow-up for a certain period. On September 26 2016, after the patient felt a sense of discomfort in the chest approximately from 3:00 to 8:00 a.m., he visited another clinic. He was referred to our institution on the same date due to a suspicion of ischemic heart disease (IHD). His chest pain was disappeared around 8:00-9:00 a.m.

Because of the confirmation of increase in ST for V2-4 and ventricular tachycardia (VT) in electrocardiogram (ECG), he received emergency coronary angiography (emCAG) due to a suspicion of ST-segment elevation myocardial infarction (STEMI). $<$ Coronary angiography (CAG) >: \#1 60, \#3 10, \#4AV 60, \#7AD 50, \#9 20/70, $\# 1230$, and ejection fraction (EF) $65.5 \%$. In CAG, significant stenotic lesion was not found according to the electrocardiographic change. He was hospitalized and received treatment due to a suspicion of spasm. Moderate stenosis was found in coronary, but significant stenosis and obstructed site were not found. There was no increase in CK after blood collection.

However, since troponin was slightly increased, we conducted BMIPP-Tl myocardial scintigraphy (Figure 1) on the following day due to a possible ACS by plaque rapture caused by spasm or moderate stenotic lesion. As a result, it is assumed that there would be some sort of ischemic event when hypoperfusion with $\beta$-methyliodophenyl pentadecanoic acid (BMIPP) was found in the anterior wall and apex. Later, a clear high signal intensity was not found in T2weighted image (Figure 2) after CMR, but a high signal intensity area was found in the anterior wall by the image of Balanced SSFP with Inversion Recovery. Because a negative T-wave was detected by chest lead in ECG, the event at the time would be spam or acute coronary syndrome (ACS) event in left anterior descending coronary artery (LAD) peripheral region. Therefore, we initiated oral administration of diltiazem for spasm prevention and aspirin/statin for moderate stenotic lesion (Figure 3) (Figure 4). ${ }^{1,2}$ 


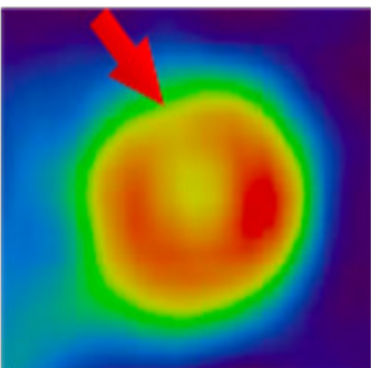

Figure I BMIPP.

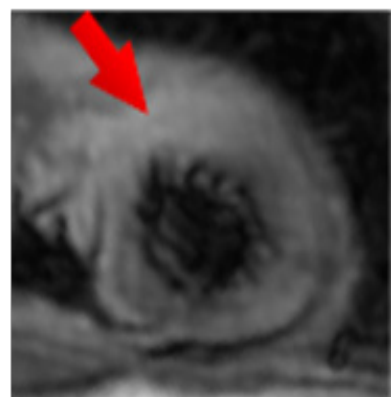

Figure 2 T2weight image.

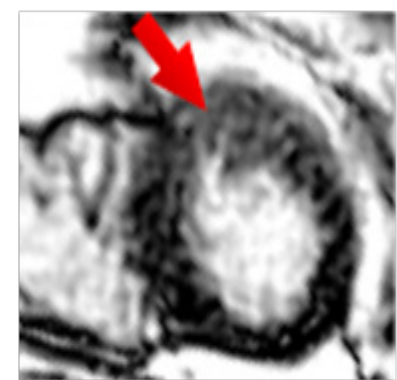

Figure 3 IR+Balanced SSFP.

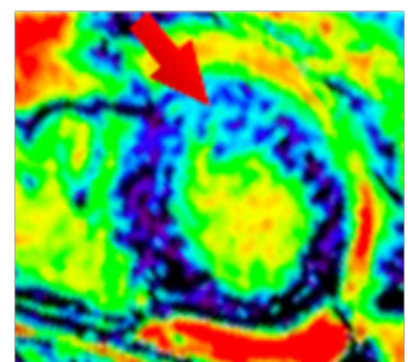

Figure $4 \mathrm{IR}+$ Balanced SSFP color.

\section{Discussion}

The sequence used this time is not a totally new method technologically. Balanced SSFP as a sequence with a capability of high-speed imaging has a high slew rate for gradient magnetic field and a high magnetic homogeneity. Compared to the MRI image equipment sold within 10years, it is considered that the imaging method proposed in the present study could satisfactorily work. Balanced SSFP has some characteristics; less artifact by movement to collect an extremely short TR/TE and a long T2 value (i.e. water) as equivalent to a high signal intensity with contrast T2/T1. In this manner, it may possibly be similar to T2-weighted image and compensate for the shortcoming, but myocardial signal intensity could be too high if using such method as it is..$^{3-6}$

The solution for the short coming is to use Inversion Recovery simultaneously and set a parameter to be a black (low signal) or null point for myocardium. Because T1 value for myocardium is approximately $1200 \mathrm{~ms}$, null point will be approximately $840 \mathrm{~ms}$ $(1200 \times 0.69)$. In addition, as a specific setting method, data collection is also set as single shot for avoiding any influence of arrhythmia and breathing.

On the other hand, in comparison with normal myocardium, impaired myocardium would have an extended T2 value by increase in extracellular fluid compartment ( $-70 \mathrm{~ms})$ and indicate a high signal intensity as similar to a normal T2-weighted image. It is considered that an extension for $\mathrm{T} 2$ value could be small due to an extremely mild ischemia in the present case. For this reason, it was difficult to make a visualization in T2-weighted image. The diagnostic imaging was confirmed after conducting the scintigraphy with use of BMIPP before CMR, but it is assumed that non-invasive diagnosis could have been conducted if performing a process of imaging by Balanced SSFP with Inversion Recovery.

\section{Acknowledgements}

None.

\section{Conflict of interest}

Author declares that there is no conflict of interest.

\section{References}

1. Kellman P, Aletras AH, Mancini C, et al. T2-prepared SSFP improves diagnostic confidence in edema imaging in acute myocardial infarction compared to turbo spin echo. Magn Reson Med. 2007;57(5):891-897.

2. Andreas Kumar, Nirat Beohar, Jain Mangalathu Arumana, et al. CMR Imaging of Edema in Myocardial Infarction Using Cine Balanced Steady-State Free Precession. JACC Cardiovasc Imaging. 2011;4(12):1265-1273.

3. Weber OM, Speier P, Scheffler K, et al. Assessment of magnetization transfer effects in myocardial tissue using balanced steady-state free precession (bSSFP) cine MRI. Magn Reson Med. 2009;62(3):699-705.

4. Aletras AH, Tilak GS, Natanzon A, et al. Retrospective determination of the area at risk for reperfused acute myocardial infarction with T2-weighted cardiac magnetic resonance imaging: histopathological and displacement encoding with stimulated echoes (DENSE) functional validations. Circulation. 2006;113(15):1865-1870.

5. Abdel-Aty H, Zagrosek A, Schulz-Menger J, et al. Delayed enhancement and T2-weighted cardiovascular magnetic resonance imaging differentiate acute from chronic myocardial infarction. Circulation. 2004;109(20):2411-2416.

6. Ify Mordi, David Carrick, Hiram Bezerra, et al. T1 and T2 mapping for early diagnosis of dilated non-ischaemic cardiomyopathy in middle-aged patients and differentiation from normal physiological adaptation You have access. European Heart Journal. 2015. p. 797-803.

7. Dominik Paul, Michael Markl, Hans-Peter Fautz, et al. T2-Weighted Balanced SSFP Imaging (T2-TIDE) Using Variable Flip Angles. Magn Reson Med. 2006;56(1):82-93. 